\title{
Correlation of Breast Cancer Subgroups and Axillary Metastases with 18F-FDG PET/CT and the Contribution of 18F-FDG PET/CT in the Management of the Axilla
}

\author{
Nazmi Ozer and Abdullah Sahin \\ Department of General Surgery, University of Health Sciences, Adana City Training and Research Hospital, Turkey
}

\begin{abstract}
Objective: To investigate whether breast and axilla maximum standard uptake (SUVmax) values contribute to the treatment approach in breast cancer subgroups.

Study Design: A descriptive study.

Place and Duration of Study: University of Health Sciences/Adana City Training and Research Hospital, Turkey, from April 2017 to September 2019.

Methodology: Ninety patients, operated for early breast cancer, were examined histopathologically and demographically. Those patients were divided into subgroups, according to the St. Gallen consensus. Breast and axillary SUVmax uptakes of these subgroups were determined using (Fluorine ${ }^{18}$ fluorodeoxyglucose positron emission tomography/computed tomography (18F-FDG PET/CT). These values were compared with postoperative pathology results

Results: The triple negative group had the highest values and the Luminal B pos $(+)$ group had the lowest values, when the mean values of breast and axilla SUVmax were compared. Axilla SUVmax value in those with metastatic lymph node was 1.939 times higher than those without it. The cut-off value of SUVmax value of the axillary lymph nodes was found to be 1.1 in distinguishing metastatic from non-metastatic.

Conclusion: As the biological aggressiveness of tumor increased, SUVmax values increased in parallel. As a result, SUVmax values guided in determining the presence of axillary metastasis and treatment strategy.
\end{abstract}

Key Words: Breast cancer, 18F-FDG PET/CT, SUVmax values, Tumor subgroups, Axilla.

How to cite this article: Ozer N, Sahin A. Correlation of Breast Cancer Subgroups and Axillary Metastases with 18F-FDG PET/CT and the Contribution of 18F-FDG PET/CT in the Management of the Axilla. J Coll Physicians Surg Pak 2021; 31(02):150-155.

\section{INTRODUCTION}

Today, breast cancer is diagnosed at an early stage as methods of diagnosis and screening have become widespread and easily accessible; and its treatment gives good results. The diagnosis and staging of breast cancer are very important in determining the optimal treatment option for the patient. Tumor size, axillary lymph node and distant metastases, estrogen receptor (ER), progesterone receptor (PR), Ki 67 proliferation index, status of human epidermal growth factor receptor 2 (HER 2), positivity of ecadherin, status of lymphovascular, and perineural invasions are determinative in terms of staging, treatment planning and prognosis. ${ }^{1}$ Moreover, grade, localisation of tumor and genetic mutations are the guiding factors in the treatment. ${ }^{2}$

Correspondence to: Dr. Nazmi Ozer, Department of General Surgery, University of Health Sciences, Adana City Training and Research Hospital, Turkey

E-mail: nazmiozer83@gmail.com

Received: July 28, 2020; Revised: September 24, 2020;

Accepted: February 02, 2021

DOI: https://doi.org/10.29271/jcpsp.2021.02.150
Florine $^{18}$-fluorodeoxyglucosepositronemissiontomography/computed tomography (18F-FDG PET / CT) is routinely used in the staging of breast cancer, in relapse follow-up, in determining the response to neo-adjuvant, and adjuvant therapy. ${ }^{3}$ This imaging method utilises the metabolic high glycolytic activities of tumor cells. Many parameters are there, such as total lesion glycolysis (TLG), metabolic tumor volume (MTV) and tumor/liver uptake ratio (TLR) in determining tumor metabolism clinicopathologically, but the most frequently used parameter is the maximum standard uptake value (SUVmax). ${ }^{4}$

Breast cancer has been subdivided according to molecular types, because of thetumoral heterogeneity. These subgroups are determined on the basis of immunohistochemical parameters and proliferation index according to the St. Gallen consensus into Luminal A, Luminal B (-) negative, Luminal B (+) positive, Human epidermal growth factor receptor 2 (HER 2) (+) positive, and Triple negative. $^{5}$

E-cadherin is an important intercellular adhesion molecule. The result of its mutation is responsible for non-contact and weak contact unicellular tumor migration. ${ }^{6}$ Loss of e-cadherin is traditionally associated with promoting enhanced cancer invasion and single cell invasion. ${ }^{7}$ 
The aim of this study was to examine whether the 18F-FDG PET/CT examination could be a guide for axillary metastasis in breastcancersubgroups.

\section{METHODOLOGY}

A preoperative 18F-FDG PET / CT scan was performed on 90 patients, who were operated for early stage breast cancer, between April 2017 and September 2019 at University of Health Sciences/Adana City Training and Research Hospital, Turkey. Breast and axilla SUVmax values determined by this imaging method were evaluated in comparison with the clinic pathological features and postoperative pathology results in tumor subgroups.

Clinical information and pathology results were obtained from follow-up files and hospital information system. The patients were recorded separately according to age, tumor size, laterality and localisation. The grade of tumor was determined with Modified Scarf-Bloom-Richardson staging system. Immunohistochemically, ER (estrogen receptor), PR (progesterone receptor), HER 2 (human epidermal growth factor receptor 2), and Ki 67 proliferation index were used to separate tumor subgroups (Figure 1). In addition, e-cadherin mutation, Iymphovascular invasion and perineural invasion were examined in tumor subgroups in pathological examination for estrogen receptor (ER) and progesterone receptor (PR) determination, if there was at least $1 \%$ positive tumor nucleus in the tested sample, the receptor was considered positive. ${ }^{8}$

HER 2 was evaluated immunohistochemically and scored as 0 , $+1,+2,+3$ according to the staining intensity. Those with score $0,+1$ were considered negative while those with score +3 were considered positive. In those with score +2 , gene amplification was performed by using fluorescence in situ hybridisation (FISH) technique. HER 2 was considered positive, if the ratio of HER 2 gene copies to chromosome 17 was found to be higher than 2.2 of the 17 signal. ${ }^{9}$ According to St. Gallen consensus, if the Ki 67 proliferation index was below 14\%, it was considered to be low, and if it was $14 \%$ or above, it was considered to be a high proliferation index. ${ }^{5}$

Those who were mutated for e- cadherin were considered positive, and those who had not mutated were considered negative. In addition, the status of lymphovascular and perineural invasion was examined pathologically. Those with invasion were evaluated as positive, and those without invasion as negative.

Modified radical mastectomy or breast conserving surgery + sentinel lymph node biopsy were applied to the patients as a type of surgery. The type of surgery was determined according to factors such as the patient's age, additional diseases, whether he wanted to receive postoperative radiotherapy, breast/tumorsize and preference.

For 18F-FDG PET / CT imaging, the oral intake of the patients was stopped after 24:00 at night. They were asked to be in a fasting state for eight hours. Patients with blood glucose above $200 \mathrm{mg} /$ $\mathrm{dl}$ were declined the imaging. After one hour of intravenous injection of 18 F-FDG in an amount of $9.97 \mathrm{mCl}$ (369 MBq), imaging was performed with Philips Ingeuity TF brand 16 slice spiral PET CT device, using time-of-flight (TOF) technology. Cranio-caudal whole body scans were made in three dimensions, and axial, coronal and sagittal sections were taken.The images were examined by thinning up to $2 \mathrm{~mm}$, using XSHARP technology and Richardson-Lucy maximum likelihood algorithm.

Images were evaluated by a single nuclear medicine professional, and areas of abnormal activity were recorded. SUVmax values were calculated using the formula - the measured activity concentration $[\mathrm{Bq} / \mathrm{mL}] \times$ body weight $[\mathrm{kg}] /$ injected activity $[\mathrm{Bq}]$.

Statistical package for the social sciences (SPSS) version 21 programme was used. Normality control of continuous variables was performed with the Shapiro-Wilk test. Quantitative data were expressed as Mean \pm S.D; and qualitative data as frequencies and percentages. Student's t-test was used to compare means of breast and axilla SUVmax, while unilateral variance analysis (UVA) was used to compare means of breast and axilla SUVmax in tumor subgroups, and Tukey was used in post hoc tests. Logistic regression analysis (LRA) was used to determine how many times the mean of axilla SUVmax was higher in those with metastatic lymph node than those without it, which was expressed by odds ratio. Chi-square test was used to find the the relationship of grade, e-cadherin, lymphovascular invasion and perineural invasion, with tumor subgroups. Axilla SUVmax cut-off value was determined for metastatic lymph node with Medcalc 16 package programme. In all analyses, $p<0.05$ were considered as statisticaly significant.

\section{RESULTS}

All the patients were females, the mean age of the patients was $54.72 \pm 14.15$, ranging from $29-82$ years, and the mean tumor size was $20.24 \pm 9.13 \mathrm{~mm}(5-37 \mathrm{~mm})$. The tumor was seen in right breast in 39 patient (43.3\%), and in left breast in 51 patients (56.7\%). In the evaluation of the quadrants, $60 \%$ patients were in the upper outer quadrant ( $n=54), 23.3 \%$ in the upper inner quadrant $(n=21), 8.9 \%$ in the lowerinnerquadrant $(n=8), 4.4 \%$ in the lower outer quadrant $(n=4)$, and $3.3 \%$ patients had tumors in the retroareolar region. When the histopathological type of the tumor was examined, $78.9 \%$ of invasive ductal carcinoma ( $n=71), 14.4 \%$ of invasive lobular carcinoma ( $n=13)$, and $6.7 \%$ of medullary carcinoma $(n=6)$ were seen. The most common subgroups were Luminal B neg (-) $31.1 \%(n=28)$ and Luminal A 28.9\% $(n=26)$; while the rarest groups were triple negative $13.3 \%(n=12)$ and HER $2(+)$ $11.1 \%(n=10)$.

The patients were performed 2 types of operations as MRM (modified radical mastectomy) and BCS+SLNB (breast-conserving surgery + sentinel lymph node biopsy). While MRM ( $n=74$ ) operation was performed in $82.2 \%$ of the patients, BCS+SLNB $(n=16)$ operation was performed in $17.8 \%$ of patients. 


\begin{tabular}{|c|c|c|c|c|c|c|c|c|c|c|c|c|c|}
\hline & \multicolumn{2}{|c|}{ Luminal A } & \multicolumn{2}{|c|}{ Luminal B- } & \multicolumn{2}{|c|}{ Luminal B+ } & \multicolumn{2}{|c|}{ HER 2+ } & \multicolumn{2}{|c|}{ Triple Negative } & \multicolumn{2}{|c|}{ Total } & \multirow[b]{2}{*}{$p$} \\
\hline & $\mathbf{n}$ & $\%$ & $\mathbf{n}$ & $\%$ & $\mathbf{n}$ & $\%$ & $n$ & $\%$ & $\mathbf{n}$ & $\%$ & $n$ & $\%$ & \\
\hline \multicolumn{14}{|l|}{ Grade } \\
\hline 1 & 4 & 15.4 & 0 & 0.0 & 2 & 14.3 & 1 & 10 & 0 & 0.0 & 8 & 8.9 & \multirow{3}{*}{$<0.001$} \\
\hline 2 & 19 & 73.1 & 24 & 85.7 & 12 & 85.7 & 8 & 80 & 4 & 33.3 & 67 & 74.4 & \\
\hline 3 & 3 & 11.5 & 4 & 14.3 & 0 & 0.0 & 1 & 10 & 8 & 66.7 & 15 & 16.7 & \\
\hline \multicolumn{14}{|c|}{ E-Cadherin } \\
\hline Positive & 19 & 73.1 & 16 & 57.1 & 14 & 100.0 & 10 & 100.0 & 8 & 66.7 & 67 & 74.4 & \multirow{2}{*}{0.011} \\
\hline Negative & 7 & 26.9 & 12 & 42.9 & 0 & 0.0 & 0 & 0.0 & 4 & 33.3 & 23 & 25.6 & \\
\hline \multicolumn{14}{|c|}{ Lymphovascular invasion } \\
\hline Absent & 7 & 26.9 & 12 & 42.9 & 12 & 85.7 & 7 & 70.0 & 4 & 33.3 & 42 & 46.7 & \multirow{2}{*}{0.003} \\
\hline Present & 19 & 73.1 & 16 & 57.1 & 2 & 14.3 & 3 & 30.0 & 8 & 66.7 & 48 & 53.3 & \\
\hline \multicolumn{14}{|c|}{ Perineural invasion } \\
\hline Absent & 18 & 69.2 & 20 & 71.4 & 14 & 100.0 & 10 & 100.0 & 8 & 66.7 & 70 & 77.8 & \multirow{2}{*}{0.049} \\
\hline Present & 8 & 30.8 & 8 & 28.6 & 0 & 0.0 & 0 & 0.0 & 4 & 33.3 & 20 & 22.2 & \\
\hline
\end{tabular}

Table II: Evaluation of tumor subgroups according to the average involvement of breast and axilla SUVmax after 18f-FDG PET/CT.
\begin{tabular}{|l|l|c|c|}
\hline SUVmax-localization & Group & Mean \pm SD & Min-Max \\
\hline \multirow{5}{*}{ Breast SUVmax } & Luminal A & $4.24 \pm 1.71$ & $2.66-7.01$ \\
\cline { 2 - 4 } & Luminal B- & $7.08 \pm 2.78$ & $3.15-10.50$ \\
\cline { 2 - 4 } & Luminal B+ & $4.05 \pm 3.54$ & $0.91-10.50$ \\
\cline { 2 - 4 } & Her2+ & $5.17 \pm 3.59$ & $1.54-10.50$ \\
\cline { 2 - 4 } & Triple Negative & $10.95 \pm 0.41$ & $10.4-11.30$ \\
\cline { 2 - 4 } & Total & $6.09 \pm 3.4$ & $0.91-11.30$ \\
\hline \multirow{5}{*}{ Axilla SUVmax } & Luminal A & $2.35 \pm 1.75$ & $0.00-5.400$ \\
\cline { 2 - 4 } & Luminal B- & $1.24 \pm 0.95$ & $0.00-2.63$ \\
\cline { 2 - 4 } & Luminal B+ & $1.21 \pm 0.80$ & $0.00-2.50$ \\
\cline { 2 - 4 } & Her2+ & $1.85 \pm 0.70$ & $0.00-2.50$ \\
\cline { 2 - 4 } & Triple Negative & $2.91 \pm 2.74$ & $0.70-9.00$ \\
\cline { 2 - 4 } & Total & $1.73 \pm 1.65$ & $0.00-9.00$ \\
\hline p: One-way analysis of variance & & \\
\end{tabular}

Table III: The comparison of tumor subgroups according to positivity/negativity rates of axillary lymph node.

\begin{tabular}{|c|c|c|c|c|c|c|c|c|c|c|c|c|c|}
\hline \multirow[t]{2}{*}{ Tumor subgroups } & \multicolumn{2}{|c|}{ Luminal A } & \multicolumn{2}{|c|}{ Luminal B- } & \multicolumn{2}{|c|}{ Luminal B+ } & \multicolumn{2}{|c|}{ Her2+ } & \multicolumn{2}{|c|}{ Triple Negative } & \multicolumn{2}{|c|}{ Total } & \multirow[b]{2}{*}{$p$} \\
\hline & $\mathbf{n}$ & $\%$ & $\mathbf{n}$ & $\%$ & $\mathbf{n}$ & $\%$ & $\mathbf{n}$ & $\%$ & $\mathbf{n}$ & $\%$ & $\mathbf{n}$ & $\%$ & \\
\hline \multicolumn{14}{|c|}{ Metastatic Lymph Node } \\
\hline Absent & 4 & 15.4 & 16 & 57.1 & 10 & 71.4 & 5 & 50.0 & 1 & 8.3 & 36 & 40.0 & \multirow{2}{*}{$<0.001$} \\
\hline Present & 22 & 84.6 & 12 & 42.9 & 4 & 28.6 & 5 & 50.0 & 11 & 91.7 & 54 & 60.0 & \\
\hline
\end{tabular}

While an average of $5.25 \pm 0.45$ lymph nodes were removed from the BCS + SLNB group after the operation (min-max: $5-6)$, the number of metastatic lymph nodes was found to be $0.25 \pm 0.45$. While an average of $20.54 \pm 5.21$ lymph nodes were removed in the MRM operation group (min-max: 14-31), the number of metastatic lymph nodes was found to be 1.42 \pm 1.53 . According to this result, the number of metastatic lymph nodes was found to be statistically significantly higher in patients in the MRM group, who underwent level 1-2 axillary dissection compared to the BCS + SLNB group $(p<0.001)$.

In the evaluation of tumor subgroups according to grade, ecadherin, lymphovascular invasion and perineural invasion frequency, Grade 1 tumor incidence was higher in Luminal A group (15.4\%) compared to the other groups, which was considered statistically significant. When all subgroups were evaluated, grade 2 tumors were the most common, except for the triple negative group $(74.4 \%, n=67)$. Grade 3 tumors were seen most frequently $(66.7 \%)$ in the triple negative group. There was statistically significant relationship between tumor subgroups and grade $(p<0.001)$

When the e-cadherin mutation was examined, it was seen that it was $100 \%(n=14),(n=10)$ positive in Luminal B pos $(+)$ and HER $2(+)$ groups. In Luminal A and triple negative groups, e-cadherin positivity was high $(73.1 \%, 66.7 \%)$. The group with the highest e-cadherin negativity $(42.9 \%)(n=$ 12/28) was Luminal B neg (-).

When the lymph vascular invasion rate was examined, the highest positivity rate was seen in Luminal A (73.1\%), and triple negative $(66.7 \%)$ groups; while the highest negative rate was seen in Luminal B pos (+) (85.7\%) and HER $2(+)$ $(70 \%)$ groups. There was statistically significant difference between the groups $(p=0.003)$. 


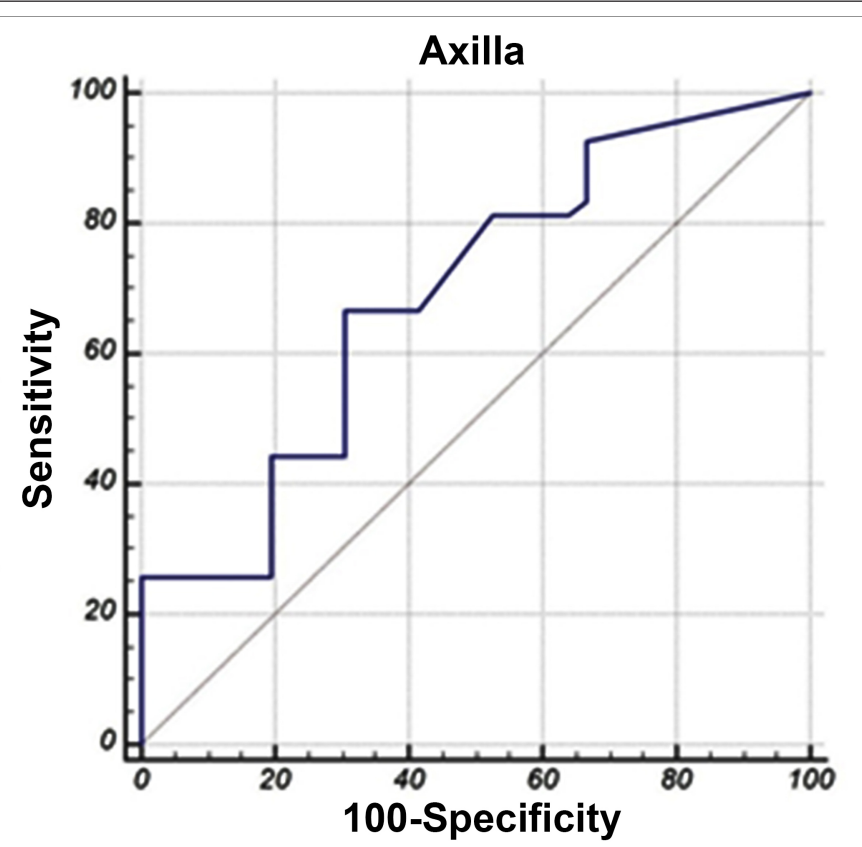

Figure 1: ROC curve analysis of axillary metastatic-nonmetastatic lymph nodes.

AUC: Area under the ROC curve.

When the perineural invasion rate is examined, the highest positivity rate was observed in the triple negative group (33.3\%); while the highest negative rate was observed in the Luminal B pos (+) and HER $2(+)$ (both $100 \%$ ) groups. When all groups were compared, it was seen that there was statistically significant difference between them $(p=0.049$, Table I).

Regarding breast SUVmax values, it was observed that the triple negative group had the highest values $(10.95 \pm 0.41)$ and the Luminal B pos $(+)$ group had the lowest values (4.05 $\pm 3.54, p<0.001$ ).

When axilla SUVmax values were compared, it was observed that the triple negative group had the highest values (2.91 \pm 2.74), and Luminal B neg (-) and Luminal B pos (+) groups had the lowest values, respectively $(1.24 \pm 0.95$ vs. $1.21 \pm$ 0.80 ). There was statistically significant difference between the groups ( $p=0.001$, Table II).

When tumor subgroups were compared according to the positivity/negativity rates of axillary lymph node, the highest rate of positivity was observed in the triple negative group $(91.7 \%, n=11 / 12)$. The highest rate of negativity was observed in Luminal B pos (+) group $(71.4 \%, n=10 / 14)$. There was statistically significant difference between the groups ( $p<0.001$, Table III). High SUVmax values, presence of metastatic lymph node, and aggressiveness of tumor subgroups were observed in direct proportion.

The mean axilla SUVmax values was found to be higher in patients with metastatic lymph nodes compared to those without metastatic lymph nodes $(p=0.001)$. Axilla SUVmax value was 1.939 times higher in those with metastatic lymph nodes compared to those without metastatic lymph nodes.
The power of axilla SUVmax value in separating metastatic lymph node was determined as $69.1 \%$, which was statistically significant $(p=0.001)$. When the axilla SUVmax value was above 1.1, it successfully distinguished the metastasised lymph nodes at a rate of $66.67 \%$ and the non-metastatic lymph nodes with $69.44 \%$ (Figure 1 ).

\section{DISCUSSION}

Breast cancer is one of the malignancy types, in which tumoral heterogeneity is most common. This is because there are many factors that affect tumor behaviour. ${ }^{3}$ Therefore, tumor subgroups were formed and it was observed that the behaviour, response to treatment, and prognosis of each group were different. ${ }^{5}$ Today, 18F-FDG PET / CT is used in many tumor types in terms of tumor metabolism and staging, and guides the clinician in subjects such as metastasis, recurrence follow-up, and response to treatment in breast cancer cases. The extent of axillary surgery is still determined by preoperative and intraoperative lymph node biopsies.

The aim of this study was to evaluate the tumor subgroups, to determine the SUVmax values of metastatic and non-metastatic lymph nodes by comparing with postoperative pathology results, and to determine the SUVmax values, above which metastasis may be identified; and whether these values can be standardised and axilla management can be done accordingly.

Considering the present results - the facts that the tumor was located in the left breast and upper outer quadrant, and the histopathological type of invasive ductal carcinoma, correlated well with the reported literature. ${ }^{10,11}$ Tumor subgroups were determined according to the St. Gallen consensus; and the most common group was Luminal B neg(-), the rarest groups was HER $2(+)$ and triple negative. It was obtained similar findings in the study by Önner et al. ${ }^{12}$

Two types of surgery were applied to the patients: modified radical mastectomy (MRM) and breast conserving surgery with sentinel lymph node biopsy (BCS + SLNB). When the axillary lymph nodes were examined, greater number positivity of axillary lymph nodes was statistically significant difference in patients, who underwent MRM. The authors believe that this is due to the frozen examination of patients, who underwent BCS + SLNB, and more clear results were obtained in the pathological examinations performed in paraffin sections after MRM. ${ }^{11}$

When the tumors were analysed according to their grade, it is observed that grade 1 tumors were most frequently in the Luminal A group, and grade 3 tumors were most frequently in the triple negative group. Grade 2 tumors were most common in all groups. It was obtained similar results in the study on 583 patients by Wang et al. ${ }^{13}$

While the positivity of e-cadherin was in the highest rate $(100 \%)$ in Luminal B pos (+) group, the negativity of it was 
observed in the Luminal B neg (-) group with the highest rate $(42.9 \%)$. In the studies, it was observed that high rate of capsule invasion in the CC genotype of e-cadherin, while it was observed a high rate of perinodal invasion in the CA genotype of it. ${ }^{14}$

When the status of lymphovascular and perineural invasions were examined, the high positivity of the triple negative group is noteworthy and Luminal B pos $(+)$ group has high negativity rates, in both statuses. Perineural and lymphovascular invasions were one of the malignant prognostic parameters. Lymphatic invasion increases the risk for relapse; whereas, blood vessel invasion indicates the possibility of distant metastasis. ${ }^{15}$

When the rates of metastatic lymph node was examined, the highest rate of positivity was observed in the triple negative group $(91.7 \%)$; whereas, the highest rate of negativity was observed in Luminal B pos (+) group (71.4\%). In addition, when the breast and axilla SUVmax values were examined, the highest SUVmax values in the breast was observed in the triple negative $(10.95 \pm 0.41)$ group, while the lowest SUVmax values was observed in the Luminal B pos (+) $(4.05$ \pm 3.54 ) group. When it was examined the SUVmax values in the axilla, the highest values were observed in the triple negative $(2.91 \pm 2.74)$ group, while the lowest values were observed in the Luminal B (-) $(1.24 \pm 0.95)$ and Luminal B pos $(+)(1.21 \pm 0.80)$ group. It is clearly seen that the highness in SUVmax values is directly proportional to axillary metastasis rates. In addition, the higher the grade, lymphovascular and perineural invasion rates of the tumor, the higher the rate of metastasis and SUVmax values in the breast and axilla.

Studies have shown that Luminal A group has low expression in cellular proliferation genes; and it has been reported that Luminal $B$ groups have more aggressive and malignant prognosis than Luminal A. However, in this study, except grade parameter, Luminal B group was observed to be less aggressive than Luminal A group, when the lymphovascular, perineural invasion parameters, axillary metastatic lymph node ratio and SUVmax values were examined. The HER 2 gene plays a role in tumor growth and progression, and HER $2(+)$ group is associated with high rates of recurrence and mortality. ${ }^{16}$ It was observed that the triple negative group is more aggressive than other groups; and has worse survival results. ${ }^{17}$

In addition, it was showed that the triple negative group has a higher 18F-FDG uptake compared to Luminal A and B groups ${ }^{18,19}$ The study carried out by Kajary et al. has showed that subtypes of biological tumor have a significant relationship with other volumetric parameters except MTV (metabolic tumor volume). ${ }^{20}$

Considering the limitation of this study, although we looked at the metastatic lymph node positivity and negativity in tumor subgroups, it was not examined how many lymph nodes were excised in which subgroup and how many of the excised lymph nodes were positive.

The mean axilla SUVmax was found to be higher and statistically significant in patients with metastatic lymph nodes than those without metastatic lymph nodes $(p=0.001)$. Accordingly, the axillary SUVmax value was 1.939 times higher in patients with metastatic lymph nodes than those without metastatic lymph nodes. The cut-off value of SUVmax value of axillary lymph nodes in distinguishing between metastatic and non-metastatic lymph nodes was $>1.1$. The low number of patients in the study caused no significant results for axillary metastasis when SUVmax values of subgroups were examined individually. Because of the specificity and sensitivity rates are not very high, tumor biology and risk factors also need to be considered. Standardisation can be achieved for subgroup at what values the decision of axillary dissection will be performed, from larger patient series and meta-analyses of the studies on this subject.

\section{CONCLUSION}

SUVmax values increased in parallel with the increasing biological tumor aggression. In metastatic axillary lymph nodes, SUVmax values were approximately two times higher than nonmetastatic lymph nodes. If the SUVmax value is above 1.1, the clinician should be alerted for metastasis.

\section{ETHICAL APPROVAL:}

With the approval of the decision 42/578 of Clinical research ethics committee of Adana City Training and Research Hospital of 23 October 2019. All procedures performed in studies involving human participants were in accordance with the ethical standards of the institutional and/or national research committee and with the 1964 Helsinki declaration and its later amendments or comparable ethical standards.

\section{PATIENTS' CONSENT:}

Consent for the participation in study was not obtained from patients as data was collected from medical record without disclosing the indentity of participants.

\section{CONFLICT OF INTEREST:}

The authored declared no conflict of interest.

\section{AUTHORS' CONTRIBUTION:}

NO, AS: Concept, Design, supervision, data collection and processing, interpretation, literature search, writing manuscript, critical review.

\section{REFERENCES}

1. André S, Pereira T, Silva F, Machado P, Vaz F, Aparício M, et al. Male breast cancer: Specific biological characteristics and survival in a Portuguese cohort. Molecular Clini Oncol 2019; 10(6):644-54. doi: 10.3892/mco.2019.1841. 
2. Feng Y, Spezia M, Huang S, Yuan C, Zeng Z, Zhang L, et al. Breast cancer development and progression: Risk factors, cancer stem cells, signaling pathways, genomics, and molecular pathogenesis. Genes \& diseases 2018; 5(2):77-106. doi: 10.1016/j.gendis.2018.05.001.

3. Gajjala SR, Hulikal N, Kadiyala S, Kottu R, Kalawat T. Whole-body ${ }^{18} \mathrm{~F}$-fluorodeoxyglucose positron emission tomography-computed tomography $\left({ }^{18} \mathrm{~F}-\mathrm{FDG} \mathrm{PET} / \mathrm{CT}\right)$ for staging locally advanced breast cancer: A prospective study from a tertiary cancer centre in south India. Indian J Med Res 2018; 147(3):256-62. doi: 10.4103/ijmr.IJMR_1368_16.

4. Kaida H, Toh U, Hayakawa M, Hattori S, Fujii T, Kurata S. et al. The relationship between 18 F-FDG metabolic volumetric parameters and clinicopathological factors of breast cancer. Nuclear Medicine Communications 2013; 34(6):562-70. doi: 10.1097/MNM.0b013e328360d945.

5. Goldhirsch A, Wood WC, Coates AS, Gelber RD, Thürlimann B, Senn HJ, et al. Strategies for subtypes - dealing with the diversity of breast cancer: Highlights of the St gallen international expert consensus on the primary therapy of early breast cancer 2011. Annals Oncol 2011; 22(8):1736-47. doi: 10.1093/annonc/mdr304.

6. Friedi $\mathrm{P}$, Alexander S. Cancer Invasion and the microenvironment: Plasticity and reciprocity. Cell 2011; 147(5): 992-1009. doi: 10.1016/j.cell.2011.11.016.

7. Horne HN, Hannah OH, Sherman ME, Palakal M, Hewitt SM, Schmidt MK, et al. E-cadherin breast tumor expression, risk factors and survival: Pooled analysis of 5,933 cases from 12 studies in the breast cancer association consortium. Sci Rep 2018; 8(1):6574. doi: 10.1038/s41598-018-23733-4.

8. Hammond MEH, Hayes DF, Dowsett M, Allred DC, Hagerty $\mathrm{KL}$, Badve S, et al. American society of clinical oncology/college of american pathologists guideline recommendations for immunohistochemical testing of estrogen and progesterone receptors in breast cancer (unabridged version). Archives Opathol Labor Med 2010; 134(7):e48e72. doi: 10.1043/1543-2165-134.7.e48.

9. Wolff AC, Hammond MEH, Schwartz JN, Hagerty KL, Allred DC, Cote RJ, et al. American society of clinical oncology/college of american pathologists guideline recommendations for human epidermal growth factor receptor 2 testing in breast cancer. Archives Pathol Labor Med 2007; 131(1): 18-43. doi: 10.1200/JCO.2013.50.9984.

10. Chan S, Chen JH, Li S, Chang R, Yeh DC, Chang RF, et al. Evaluation of the association between quantitative mammographic density and breast cancer occurred in different quadrants. BMC Cancer 2017; 17(1):274. doi: 10.1186/ s12885-017-3270-0.
11. Weaver DL. Pathology evaluation of sentinel lymph nodes in breast cancer: Protocol recommendations and rationale. Mod Pathol 2010; 23(Suppl 2):p 26-32. doi: 10.1038/modpathol.2010.36.

12. Önner H, Canaz F, Dinçer M, Isiksoy S, Sivrikoz IA, Entok E, et al. Which of the fluorine-18 fluorodeoxyglucose positron emission tomography/computerised tomography parameters are better associated with prognostic factors in breast cancer? Medicine 2019; 98(22):e15925. Doi.10.1097/ MD.0000000000015925.

13. Wang J, Luo J, Jin K, Wang X, Yang Z, Ma J. et al. Biological subtype predicts locoregional recurrence after postmastectomy radiotherapy in Chinese breast cancer patients. Cancer Medicine 2020; 9(7):2427-34. doi: 10.1002/cam4.2904.

14. Turan N, Ergen A, Arıkan S. The role of Mmp-3 And E-cadherin polymorphisms in breast tumours. Deneysel tıp araştırma enstitüsü dergisi 2017; 2:2-4 p:30-37.

15. Rosai J. In Rosai and Ackerman's Surgical Pathology, Ninth edition, Vol. 2, London: Mosby, 2004; 1763-1876.

16. Gianni L, Dafni U, Gelber RD, Azambuja E, Muehlbauen S, Goldhirsch A, et al. Treatment with trastuzumab for 1 year after adjuvant chemotherapy in patients with HER2-positive early breast cancer: a 4-year follow-up of a randomised controlled trial. Lancet Oncology 2011; 12(3):236-244. doi: 10.1016/S1470-2045(11)70033-X.

17. Swisher SK, Vila J, Tucker SL, Bedrosian I, Shaitelman SF, Litton JK. et al. Locoregional control according to breast cancer subtype and response to neoadjuvant chemotherapy in breast cancer patients undergoing breast-conservingtherapy. Ann Surg Oncol 2016; 23(3):749-56. doi: 10.1245/s10434-015-4921-5.

18. Groheux D, Giacchetti S, Moretti JL, Porcher R, Espié M, Lehmann-Che J. et al. Correlation of high 18 F-FDG uptake to clinical, pathological and biological prognostic factors in breast cancer. EurJ Nuclear Medicine Molecular Imaging 2011; 38(3):426-35. doi: 10.1007/s00259-010-1640-9.

19. Dong A, Wang $Y$, Lu J, Zuo C. Spectrum of the breast lesions with increased 18F-FDG Uptake on PET/CT. Clinical Nuclear Medicine 2016; 41(7):543-57. doi: 10.1097/RLU.000000 0000001203.

20. Kajáry K, Tokés T, Dank M, Kulka J, Szakáll Jr S, Lengyel Z. Correlation of the value of 18F-FDG uptake, described by SUVmax, SUVavg, metabolic tumour volume and total lesion glycolysis, to clinicopathological prognostic factors and biological subtypes in breast cancer. Nuclear Medicine Communications 2015; 36(1):28-37. doi: 10.1097/MNM.0000000000000217. 